\title{
TITLE:
}

\section{Oscillatory motions of an active deformable particle}

\author{
$\operatorname{AUTHOR}(\mathrm{S}):$
}

Tarama, Mitsusuke; Ohta, Takao

\section{CITATION:}

Tarama, Mitsusuke ...[et al]. Oscillatory motions of an active deformable particle. Physical Review E 2013, 87(6): 062912.

ISSUE DATE:

2013-06-18

URL:

http://hdl.handle.net/2433/176342

RIGHT:

(C)2013 American Physical Society 
PHYSICAL REVIEW E 87, 062912 (2013)

\title{
Oscillatory motions of an active deformable particle
}

\author{
Mitsusuke Tarama ${ }^{1,2,3,{ }^{*}}$ and Takao Ohta ${ }^{1,3,4, \dagger}$ \\ ${ }^{1}$ Department of Physics, Kyoto University, Kyoto 606-8502, Japan \\ ${ }^{2}$ Institute for Solid State Physics, University of Tokyo, Kashiwa, Chiba 277-8581, Japan \\ ${ }^{3}$ Department of Physics, University of Tokyo, Tokyo 113-0033, Japan \\ ${ }^{4}$ Soft Matter Center, Ochanomizu University, Tokyo 112-0012, Japan
}

(Received 8 April 2013; published 18 June 2013)

\begin{abstract}
We investigate dynamics of an active particle in which shape deformations occur spontaneously. In two dimensions, the deformations are expanded in terms of the Fourier series and the couplings of different modes are taken into consideration truncated up to lower orders. We focus our attention on the special symmetrical structure between the coupled equations of $n$ - and $2 n$-mode deformations for $n=1$, and those for $n=2$. We show that an oscillatory bifurcation occurs for $n=2$, which corresponds mathematically to the bifurcation for $n=1$ where a straight motion becomes unstable and a circular motion appears. At the oscillatory state, the particle undergoes either a spinning motion or a standing oscillation of shape deformations.
\end{abstract}

DOI: 10.1103/PhysRevE.87.062912

PACS number(s): 05.45.-a, 05.70.Ln, 82.40.Ck

\section{INTRODUCTION}

Shape deformation of microorganisms such as living cells and protozoa plays an important role in their active motion [1-5]. Active motions accompanied with shape deformation have also been observed in nonbiological systems such as spontaneously moving oil droplets [6-8]. In order to elucidate these phenomena from a theoretical viewpoint, one needs to develop nonlinear dynamics and nonequilibrium statistical physics. There are a number of elaborated model systems for specific biological experiments [9-11]. Simple models of self-propelled motion due to shape deformation have also been developed [12-15].

Recently, we have developed a theory of the dynamics of a deformable self-propelled particle which undergoes a spinning motion [16,17]. In fact, there are a lot of experiments of a soft particle or a living cell which undergoes a spinning motion spontaneously [4,5,18-26]. Our previous analysis was based on former works of a deformable self-propelled particle [27-30], where the velocity of center of mass and the symmetric tensor variable representing deformation are taken into consideration. In Refs. [16,17], we have additively introduced an antisymmetric tensor variable, which is directly related to an angular momentum. Therefore, the set of timeevolution equations introduced is applicable to systems for which an angular momentum can be defined [18-24]. In contrast, in some experimental studies, a spinning motion of an active soft object is related to a chemical wave inside the particle [25] or traveling waves on the interface [4,5,26], to which our previous model seems not to be applicable. The aim of this paper is to introduce and study a theoretical model for such an active spinning motion from a general view point in two dimensions where the interfacial motion plays an important role.

The organization of this paper is as follows: In the next section, we introduce the time-evolution equations for an active deformable particle where the deformation is represented by its

*tarama@scphys.kyoto-u.ac.jp

†takao@scphys.kyoto-u.ac.jp
Fourier components. We emphasize a special symmetry of the coupled equations for different-Fourier-mode deformations in Sec. III. As an example, we consider the coupled equations of the first and second mode deformations and of the second and the fourth mode deformations in Sec. IV, where a circular motion and a spinning motion are obtained, respectively. In Sec. V, we analyze the dynamics with the second and the fourth mode deformations in more detail, and this is compared with the numerical results in Sec. VI. Discussion of the results obtained is given in Sec. VII. In Appendix A, the relation between the Fourier components of deformation and its tensor descriptions is summarized. The fact that the center of mass of a particle cannot move when only the modes with even integers in the Fourier expansion are retained is described in Appendix B.

\section{MODEL}

Deformations of a particle around a circular shape with radius $R_{0}$ are written as

$$
R(\phi)=R_{0}+\delta R(\phi, t),
$$

where the deviation $\delta R$ can be expanded in the Fourier series

$$
\delta R(\phi, t)=\sum_{n=-\infty}^{\infty} c_{n}(t) e^{i n \phi} .
$$

We introduce time-evolution equations in terms of the Fourier coefficients $c_{ \pm n}$. The zeroth mode is put to be $c_{0}=0$ imposing the condition of area conservation. Rigid translational motion is treated as follows. Suppose that the center of mass $\rho$ changes for an infinitesimal time interval $\delta t$ as

$$
\delta \rho=\mathbf{v} \delta t .
$$

In two dimensions we may put this, without loss of generality, as

$$
\delta \boldsymbol{\rho}=\epsilon\left(\cos \theta_{1}, \sin \theta_{1}\right),
$$

where $\epsilon$ is the magnitude of deviation and $\theta_{1}$ is its direction. This means that $\mathbf{v}$ contains only the first mode $n= \pm 1$. We 
define $v_{ \pm 1}=(v / 2) e^{ \pm i \theta_{1}}$ so that

$$
\mathbf{v}=\left[v_{1}+v_{-1},-i\left(v_{1}-v_{-1}\right)\right] .
$$

Then, we can take $v_{ \pm 1}$ as dynamical variables removing the modes $c_{ \pm 1}$ from the expansion (2).

Hereafter, we take into consideration deformations, as well as the velocity of the center of mass $v_{ \pm 1}$, up to the fourth orders of the Fourier components: $c_{ \pm 2}, c_{ \pm 3}, c_{ \pm 4}$. We derive the time-evolution equations for these variables based on the two fundamental symmetries, uniformity and isotropy of space. The set of equations is constructed such that the translational symmetry and the rotational symmetry are satisfied. The couplings of the $v_{ \pm 1}$ modes and the deformations are considered up to the cubic nonlinearity. From these considerations, the time-evolution equations are obtained as

$$
\begin{aligned}
\frac{d v_{1}}{d t}= & -\kappa_{1} v_{1}-\alpha_{0}\left|v_{1}\right|^{2} v_{1} \\
& +\alpha_{1} c_{2} v_{-1}+\alpha_{2} c_{3} c_{-2}+\alpha_{3} c_{4} c_{-3} \\
& +\alpha_{4}\left|c_{2}\right|^{2} v_{1}+\alpha_{5}\left|c_{3}\right|^{2} v_{1}+\alpha_{6}\left|c_{4}\right|^{2} v_{1} \\
& +\alpha_{7} c_{2}^{2} c_{-3}+\alpha_{8} c_{2} c_{3} c_{-4}+\alpha_{9} v_{-1}^{2} c_{3} \\
& +\alpha_{10} v_{-1} c_{-2} c_{4},
\end{aligned}
$$

$$
\begin{aligned}
\frac{d c_{2}}{d t}= & -\kappa_{2} c_{2}-\beta_{0}\left|c_{2}\right|^{2} c_{2}+\beta_{1} v_{1}^{2}+\beta_{2} c_{3} v_{-1}+\beta_{3} c_{4} c_{-2} \\
& +\beta_{4}\left|v_{1}\right|^{2} c_{2}+\beta_{5}\left|c_{3}\right|^{2} c_{2}+\beta_{6}\left|c_{4}\right|^{2} c_{2} \\
& +\beta_{7} v_{1} c_{3} c_{-2}+\beta_{8} v_{1} c_{4} c_{-3}+\beta_{9} c_{3}^{2} c_{-4}+\beta_{10} v_{-1}^{2} c_{4},
\end{aligned}
$$

$$
\begin{aligned}
\frac{d c_{3}}{d t}= & -\kappa_{3} c_{3}-v_{0}\left|c_{3}\right|^{2} c_{3}+v_{1} v_{1} c_{2}+v_{2} c_{4} v_{-1} \\
& +v_{3}\left|v_{1}\right|^{2} c_{3}+v_{4}\left|c_{2}\right|^{2} c_{3}+v_{5}\left|c_{4}\right|^{2} c_{3} \\
& +v_{6} v_{1}^{3}+v_{7} v_{1} c_{4} c_{-2}+v_{8} v_{-1} c_{2}^{2}+v_{9} c_{2} c_{4} c_{-3} \\
\frac{d c_{4}}{d t}= & -\kappa_{4} c_{4}-\lambda_{0}\left|c_{4}\right|^{2} c_{4}+\lambda_{1} c_{2}^{2}+\lambda_{2} v_{1} c_{3} \\
& +\lambda_{3}\left|v_{1}\right|^{2} c_{4}+\lambda_{4}\left|c_{2}\right|^{2} c_{4}+\lambda_{5}\left|c_{3}\right|^{2} c_{4} \\
& +\lambda_{6} v_{1}^{2} c_{2}+\lambda_{7} v_{-1} c_{2} c_{3}+\lambda_{8} c_{3}^{2} c_{-2}
\end{aligned}
$$

Here, we have used the fact that $c_{-n}$ is the complex conjugate of $c_{n}$.

The above truncation of the Fourier modes is justified in the vicinity of a supercritical drift bifurcation where the velocity $v$ is small and $c_{n}$ is of the order of $v^{n}$ as long as a circular shape is stable in a motionless state [31]. However, we consider the situation that $\kappa_{2}$ is negative in the sections below. Therefore, the above set of equations (6)-(9) should be regarded as a phenomenological model valid for weak deformations.

\section{SPECIAL SYMMETRY}

We emphasize that there is a special symmetry among the equations of modes. The modes $n= \pm 1$ and $n= \pm 2$ constitute a set of coupled equations

$$
\begin{gathered}
\frac{d v_{1}}{d t}=-\kappa_{1} v_{1}-\alpha_{0}\left|v_{1}\right|^{2} v_{1}+\alpha_{1} c_{2} v_{-1}+\alpha_{4}\left|c_{2}\right|^{2} v_{1}, \\
\frac{d c_{2}}{d t}=-\kappa_{2} c_{2}-\beta_{0}\left|c_{2}\right|^{2} c_{2}+\beta_{1} v_{1}^{2}+\beta_{4}\left|v_{1}\right|^{2} c_{2} .
\end{gathered}
$$

In order to avoid complications, we have put $c_{3}=0=c_{4}$ in Eqs. (6)-(9) to obtain the above set of equations. Similarly, a set of coupled equations of $n= \pm 2$ and $n= \pm 4$ modes must take the following form:

$$
\begin{gathered}
\frac{d c_{2}}{d t}=-\kappa_{2} c_{2}-\beta_{0}\left|c_{2}\right|^{2} c_{2}+\beta_{3} c_{4} c_{-2}+\beta_{6}\left|c_{4}\right|^{2} c_{2}, \\
\frac{d c_{4}}{d t}=-\kappa_{4} c_{4}-\lambda_{0}\left|c_{4}\right|^{2} c_{4}+\lambda_{1} c_{2}^{2}+\lambda_{4}\left|c_{2}\right|^{2} c_{4} .
\end{gathered}
$$

Equations (10) and (11) have the same structures as Eqs. (12) and (13). This is a consequence of the possible coupling of modes between $c_{ \pm n}$ and $c_{ \pm 2 n}$ and independent of any details of the system considered.

The above fact implies that a bifurcation which exists in Eqs. (10) and (11) should also occur in Eqs. (12) and (13). It is known that a bifurcation between a straight motion and a circular motion in Eqs. (10) and (11) exists for $-\kappa_{1}>0$ and $\kappa_{2}>0$ [27]. The question is what kinds of dynamics appear in Eqs. (12) and (13) as a corresponding bifurcation, which we shall address below.

\section{CIRCULAR AND SPINNING MOTIONS}

Here, we show a consequence of the special similarity introduced in Sec. III. First, we make a brief review of the circular bifurcation in Eqs. (10) and (11). The terms with $\alpha_{4}$ and $\beta_{4}$ are ignored. We consider the situation $\kappa_{1}<0$ and $\kappa_{2}>0$, and $\beta_{0}=0$ so that the particle is self-propelling and it tends to be disk shaped when there is no coupling with the velocity. In general, we can put $v_{ \pm 1}=A_{1} e^{ \pm i \theta_{1}}$ and

$$
c_{ \pm n}=A_{n} e^{ \pm i n \theta_{n}}
$$

with $A_{n}$ and $\theta_{n}$ real. Then, Eqs. (10) and (11) can be written as

$$
\begin{gathered}
\frac{d A_{1}}{d t}=-\kappa_{1} A_{1}-\alpha_{0} A_{1}^{3}+\alpha_{1} A_{1} A_{2} \cos \left(2 \theta_{1}-2 \theta_{2}\right), \\
A_{1} \frac{d \theta_{1}}{d t}=-\alpha_{1} A_{1} A_{2} \sin \left(2 \theta_{1}-2 \theta_{2}\right), \\
\frac{d A_{2}}{d t}=-\kappa_{2} A_{2}+\beta_{1} A_{1}^{2} \cos \left(2 \theta_{1}-2 \theta_{2}\right), \\
2 A_{2} \frac{d \theta_{2}}{d t}=\beta_{1} A_{1}^{2} \sin \left(2 \theta_{1}-2 \theta_{2}\right) .
\end{gathered}
$$

From Eqs. (16) and (18), we have

$$
2 \frac{d \psi}{d t}=-\left(2 \alpha_{1} A_{2}+\frac{\beta_{1} A_{1}^{2}}{A_{2}}\right) \sin (2 \psi),
$$

where $\psi=\theta_{1}-\theta_{2}$.

Equation (5) gives us up to the first order of deformations

$$
\left(v_{x}, v_{y}\right)=\left(2 A_{1} \cos \theta_{1}, 2 A_{1} \sin \theta_{1}\right) .
$$

The steady straight motion is given by $\psi=0$ for longitudinal configuration and $\psi=\pi / 2$ for transverse configuration. That is, the particle is elongated parallel (perpendicular) to the migration velocity for the longitudinal (transverse) configuration. The amplitudes are given by $\kappa_{2} A_{2}=\beta_{1} A_{1}^{2} \cos 2 \psi$ and $\alpha_{0} A_{1}^{2}=-\kappa_{1}+\alpha_{1} A_{2} \cos 2 \psi$. This solution becomes unstable when the coefficient in front of $\sin 2 \psi$ in Eq. (19) changes sign. Substituting the expressions of $A_{1}$ and $A_{2}$ gives us the 
stability threshold [27]

$$
-\kappa_{1}^{c}=-\frac{\alpha_{0} \kappa_{2}^{2}}{2 \alpha_{1} \beta_{1}}+\frac{\kappa_{2}}{2} .
$$

In a similar way, we consider the corresponding bifurcation in Eqs. (12) and (13). Here, we take into consideration up to the bilinear couplings, and hence, ignore the terms with $\beta_{6}$ and $\lambda_{4}$. The time-evolution equations are given in terms of the amplitudes and the phases as

$$
\begin{gathered}
\frac{d A_{2}}{d t}=-\kappa_{2} A_{2}-\beta_{0} A_{2}^{3}+\beta_{3} A_{2} A_{4} \cos \Theta, \\
2 A_{2} \frac{d \theta_{2}}{d t}=-\beta_{3} A_{2} A_{4} \sin \Theta, \\
\frac{d A_{4}}{d t}=-\kappa_{4} A_{4}-\lambda_{0} A_{4}^{3}+\lambda_{1} A_{2}^{2} \cos \Theta, \\
4 A_{4} \frac{d \theta_{4}}{d t}=\lambda_{1} A_{2}^{2} \sin \Theta,
\end{gathered}
$$

where $\Theta=4 \theta_{2}-4 \theta_{4}$. Here, it is found from Eqs. (22)-(25) that the dynamics is governed by $A_{2}, A_{4}$, and $\Theta$. The equation for $\Theta$ is given from Eqs. (23)-(25) by

$$
\frac{d \Theta}{d t}=-\Lambda\left(A_{2}, A_{4}\right) \sin \Theta,
$$

where

$$
\Lambda\left(A_{2}, A_{4}\right)=2 \beta_{3} A_{4}+\lambda_{1} \frac{A_{2}^{2}}{A_{4}} .
$$

Now we consider the situation $\kappa_{2}<0$ and $\kappa_{4}>0$ so that the second order (i.e., elliptical) deformation occurs spontaneously whereas the forth order deformation relaxes in the absence of the interaction. We may put $\lambda_{0}=0$ for $\kappa_{4}>0$, while $\beta_{0}$ should be positive for $\kappa_{2}<0$. For simplicity, we omit the terms with the coefficients $\beta_{6}$ and $\lambda_{4}$. Then, the stationary solutions of Eqs. (22) and (24) are obtained as

$$
\begin{gathered}
A_{2}=\left\{\frac{-\kappa_{2}}{\beta_{0}-\beta_{3} \frac{\lambda_{1}}{\kappa_{4}} \cos ^{2} \Theta}\right\}^{1 / 2}, \\
A_{4}=\frac{\lambda_{1}}{\kappa_{4}} A_{2}^{2} \cos \Theta .
\end{gathered}
$$

We note from Eq. (26) that there are two solutions of $\Theta$; one is $\tan \Theta=0$, which is stable as long as $\left|\kappa_{2}\right| \lessgtr \kappa_{2}^{c}$ for $\kappa_{2}^{c} \gtrless 0$. Here, we have defined the bifurcation threshold

$$
\kappa_{2}^{c}=-\frac{\beta_{0} \kappa_{4}^{2}}{2 \beta_{3} \lambda_{1}}+\frac{\kappa_{4}}{2} .
$$

The other is

$$
\cos ^{2} \Theta=\frac{\kappa_{2}^{c}-\frac{\kappa_{4}}{2}}{\left|\kappa_{2}\right|-\frac{\kappa_{4}}{2}},
$$

whose stability condition is given by $\left|\kappa_{2}\right|>\kappa_{2}^{c}>\kappa_{4} / 2$. The last inequality and Eq. (30) imply that the bifurcation from the solution $\tan \Theta=0$ to the solution given by Eq. (31) occurs when $\beta_{3} \lambda_{1}<0$ since $\beta_{0}>0$. Here, the sign of $\cos \Theta$ is determined from Eq. (29); i.e., it is positive if $\lambda_{1}>0$ while it should be negative if $\lambda_{1}<0$. If the solution (31) is stable and hence $\sin \Theta$ takes a finite value, then, from Eqs. (23) and (25), the direction of the second and the fourth mode deformation, $2 \theta_{2}$ and $4 \theta_{4}$, change monotonically in time with their difference $\Theta=4 \theta_{2}-4 \theta_{4}$ time independent. Therefore, this solution represents a spinning motion of a particle with 2and 4-mode deformations. One can check that the bifurcation of this spinning motion given by Eq. (30) corresponds to the bifurcation of the circular motion given by Eq. (21).

\section{STANDING WAVE OF DEFORMATION}

In this section, we consider the dynamics when both the second and the fourth Fourier mode deformations, $c_{ \pm 2}$ and $c_{ \pm 4}$, may occur spontaneously; i.e., both $\kappa_{2}$ and $\kappa_{4}$ can take negative values in Eqs. (12) and (13). In this case, the terms with positive values of $\beta_{0}$ and $\lambda_{0}$ should be added to avoid a divergence.

Now, we investigate the stationary solutions of Eqs. (22), (24), and (26) and their stability. Here, note that the stationary solutions of Eq. (26) have already been obtained in the previous section, and they are given by $\sin \Theta=0$ and by $\Lambda\left(A_{2}, A_{4}\right)=0$, where $A_{2}$ and $A_{4}$ are given, respectively, from Eqs. (22) and (24) as functions of $\Theta$. Generally, the stability of the stationary solutions can be analyzed by using the linear stability matrix [32] defined by

$$
\mathcal{L}_{i j}=\frac{\partial}{\partial \mathcal{X}_{j}} \frac{d \mathcal{X}_{i}}{d t}
$$

where $\mathcal{X}_{i} \in\left\{A_{2}, A_{4}, \Theta\right\}$. For a special case, that is, for the stationary solution of Eq. (26) given by $\sin \Theta=0$, the stability of Eqs. (22) and (24) and that of Eq. (26) are decoupled with each other, and hence, we can consider them separately. The linear stability matrix of Eqs. (22) and (24) is defined by

$$
L_{i j}=\frac{\partial}{\partial A_{j}}\left(\frac{d A_{i}}{d t}\right)
$$

and the stability of the solution $\sin \Theta=0$ is given by

$$
L_{\Theta}=-\Lambda \cos \Theta<0 \text {. }
$$

When $L_{\Theta}>0$, the stationary solution of $\Theta$ can be obtained from $\Lambda=0$.

Equations (22) and (24) have a pair of trivial solutions, $A_{2}=$ $A_{4}=0$, which represents a motionless disk-shaped particle without deformations. The stability of this solution is given, from Eq. (33), by

$$
\kappa_{2}>0 \text { and } \kappa_{4}>0 .
$$

Since the magnitudes of the second and fourth mode deformation are zero, their angles $\theta_{2}$ and $\theta_{4}$ lose their meaning, and hence, it is unnecessary to consider Eq. (26) and its stability Eq. (34).

For the trivial solution $A_{2}=0$ of Eq. (22), there is another solution of Eq. (24), that is, $A_{4}^{2}=-\kappa_{4} / \lambda_{0}$, where $\kappa_{4}<0$ is required since $\lambda_{0}>0$. From the stability matrix (33), one can obtain the stability condition of the pair of solutions $A_{2}=0$ and $A_{4}=\sqrt{-\kappa_{4} / \lambda_{0}}$ as

$$
\kappa_{4}<0 \quad \text { and } \quad \kappa_{2}>\beta_{3} \sqrt{-\kappa_{4} / \lambda_{0}} \cos \Theta .
$$

This gives the stability condition of a motionless particle with 4-mode static deformation. Here, note that, although the angle $\theta_{2}$ is not defined for $A_{2}=0$, we may consider it just above the 
bifurcation where $A_{2}$ becomes finite, and hence, $\Theta=4 \theta_{2}-$ $4 \theta_{4}$ is given from Eq. (26) with $\sin \Theta=0$.

Now, nontrivial stationary solution of Eq. (22) can be obtained as

$$
A_{2}=\left\{\frac{-\kappa_{2}+\beta_{3} A_{4} \cos \Theta}{\beta_{0}}\right\}^{1 / 2} .
$$

Then, the solution $A_{4}$ of Eq. (24) satisfies

$$
\beta_{0} \lambda_{0} A_{4}^{3}+\left(\beta_{0} \kappa_{4}-\beta_{3} \lambda_{1} \cos ^{2} \Theta\right) A_{4}+\kappa_{2} \lambda_{1} \cos \Theta=0 .
$$

Since Eq. (38) is cubic with respect to $A_{4}$, the solution can be solved analytically, but we do not write down the explicit form here.

When the solution of Eq. (26) is given by $\sin \Theta=0$, the angles $\theta_{2}$ and $\theta_{4}$ are both time independent as can be seen from Eqs. (23) and (25). Therefore, the solution of Eq. (38) with Eq. (37) represents a motionless particle with 2- and 4-mode static deformations. The stability condition of this state is obtained from Eqs. (33) and (34). There are at least three possibilities of bifurcations; two of them are obtained from the $2 \times 2$ matrix $L$ given by Eq. (33) [32]. The first possibility is given by the condition that the determinant of the stability matrix becomes zero, det $L=0$, where one of the eigenvalues changes its sign from negative to positive. The second possibility is a Hopf bifurcation given by the trace of the stability matrix; i.e., $\operatorname{tr} L=0$. As long as Eq. (34) is satisfied, it is expected from Eqs. (23) and (25) that the magnitudes of the second and the fourth mode deformations undergo oscillation beyond this Hopf bifurcation threshold keeping their direction $2 \theta_{2}$ and $4 \theta_{4}$ time independent. The third possibility is given by $L_{\Theta}=0$, where the stationary solution of the angles $\sin \Theta=0$ becomes unstable. In this case, it is expected from Eqs. (23) and (25) that a spinning motion occurs above the instability threshold. In fact, for $\kappa_{4}>0$, this bifurcation corresponds to the spinning bifurcation given by $\kappa_{2}=\kappa_{2}^{c}$ where $\kappa_{2}^{c}$ has been defined by Eq. (30).

When the stationary value of $\Theta$ is given by $\Lambda=0$, the solution $A_{4}$ of Eq. (38) with Eq. (37) represents a spinning motion, where a particle with 2- and 4-mode deformations rotates around its motionless center of mass. This spinning motion is the solution described in the previous paragraph. The analytical form of this solution is obtained from Eqs. (27), (37), and (38) as

$$
\begin{gathered}
A_{2}^{2}=\frac{-2 \beta_{3}\left(2 \kappa_{2}+\kappa_{4}\right)}{4 \beta_{0} \beta_{3}-\lambda_{0} \lambda_{1}}, \\
A_{4}^{2}=\frac{\lambda_{1}\left(2 \kappa_{2}+\kappa_{4}\right)}{4 \beta_{0} \beta_{3}-\lambda_{0} \lambda_{1}}, \\
\cos \Theta=-\frac{\kappa_{2} \lambda_{0} \lambda_{1}+2 \kappa_{4} \beta_{0} \beta_{3}}{\beta_{3} \lambda_{1}\left(2 \kappa_{2}+\kappa_{4}\right)}\left\{\frac{\lambda_{1}\left(2 \kappa_{2}+\kappa_{4}\right)}{4 \beta_{0} \beta_{3}-\lambda_{0} \lambda_{1}}\right\}^{1 / 2} .
\end{gathered}
$$

The stability condition of the spinning motion is obtained from the real part of all the eigenvalues of the stability matrix $\mathcal{L}$ given by Eq. (32). The characteristic equation of $\mathcal{L}$ is written as $\lambda^{3}-I_{\mathcal{L}} \lambda^{2}+I I_{\mathcal{L}} \lambda-I I I_{\mathcal{L}}=0$, where $I_{\mathcal{L}}, I I_{\mathcal{L}}$, and $I I I_{\mathcal{L}}$ are the first, second, and third invariants of the stability matrix $\mathcal{L}$. Here, we note that the first and third invariants are nothing but the trace and the determinant, respectively, whereas the second invariant is defined by

$$
I I_{\mathcal{L}}=\frac{1}{2}\left\{(\operatorname{tr} \mathcal{L})^{2}-\operatorname{tr} \mathcal{L}^{2}\right\} .
$$

It is convenient to introduce

$$
\begin{gathered}
\mathcal{P}=-\frac{1}{9} I_{\mathcal{L}}^{2}+\frac{1}{3} I I_{\mathcal{L}}, \\
\mathcal{Q}=-\frac{1}{27} I_{\mathcal{L}}^{3}+\frac{1}{6} I_{\mathcal{L}} I I_{\mathcal{L}}-\frac{1}{2} I I I_{\mathcal{L}},
\end{gathered}
$$

with which the characteristic equation can be written by using $\lambda^{\prime}=\lambda-I_{\mathcal{L}} / 3$ as $\lambda^{\prime 3}+3 \mathcal{P} \lambda^{\prime}+2 \mathcal{Q}=0$. From a straightforward calculation, we obtain the stability limit of the spinning motion as follows: First, if $\mathcal{Q}^{2}+\mathcal{P}^{3}>0$, the spinning state becomes unstable by a pitchfork bifurcation or by a Hopf bifurcation. The pitchfork bifurcation boundary is given by

$$
\mathcal{U}_{+}+\mathcal{U}_{-}+\frac{1}{3} I_{\mathcal{L}}=0
$$

whereas the Hopf bifurcation boundary is obtained as

$$
-\frac{1}{2}\left(\mathcal{U}_{+}+\mathcal{U}_{-}\right)+\frac{1}{3} I_{\mathcal{L}}=0
$$

where

$$
\begin{aligned}
& \mathcal{U}_{+}=\left[-\mathcal{Q}+\left(\mathcal{Q}^{2}+\mathcal{P}^{3}\right)^{1 / 2}\right]^{1 / 3}, \\
& \mathcal{U}_{-}=\left[-\mathcal{Q}-\left(\mathcal{Q}^{2}+\mathcal{P}^{3}\right)^{1 / 2}\right]^{1 / 3} .
\end{aligned}
$$

Note that both $\mathcal{U}_{ \pm}$are real for $\mathcal{Q}^{2}+\mathcal{P}^{3}>0$. On the other hand, if $\mathcal{Q}^{2}+\mathcal{P}^{3}<0$, the spinning motion loses its stability by a pitchfork bifurcation given by

$$
2(-\mathcal{P})^{1 / 2} \cos \left(\frac{1}{3} \arccos \left(-\mathcal{Q}(-\mathcal{P})^{-3 / 2}\right)\right)+\frac{1}{3} I_{\mathcal{L}}=0 .
$$

To summarize, the spinning motion loses its stability by a Hopf bifurcation or by a pitchfork bifurcation. After the Hopf bifurcations, an oscillation of the magnitude of deformations for the spinning particle is expected to occur.

\section{NUMERICAL RESULTS}

In this section, we show the results of numerical simulations and compare them with the analytical results obtained in the preceding section. In order to solve Eqs. (12) and (13) with $\beta_{6}=\lambda_{4}=0$ numerically, we have varied the parameters $\kappa_{2}$ and $\kappa_{4}$ with the coupling constants fixed as $\beta_{0}=-\beta_{3}=$ $\lambda_{0}=\lambda_{1}=1$. The fourth-order Runge-Kutta method has been employed with the time increment $\delta t=10^{-3}$.

The results are summarized in Fig. 1, where the bifurcation lines obtained in Sec. V are superposed. Different symbols in Fig. 1 represent different types of solutions. The plus, the cross, and the square symbols stand for a motionless particle with a nondeformed circular shape, a statically deformed shape with only 4-mode deformation, and a statically deformed shape with both 2- and 4-mode deformations. Figure 2 displays shapes of a motionless particle with 2- and 4-mode static deformations for (a) $\kappa_{2}=0.2$ and $\kappa_{4}=-0.1$ and (b) $\kappa_{2}=-0.1$ and $\kappa_{4}=$ 0.3 , and (c) with 4-mode static-deformation for $\kappa_{2}=0.4$ and $\kappa_{4}=-0.1$.

In the region indicated by the circle, a particle with both 2- and 4-mode static deformations rotates around its center 


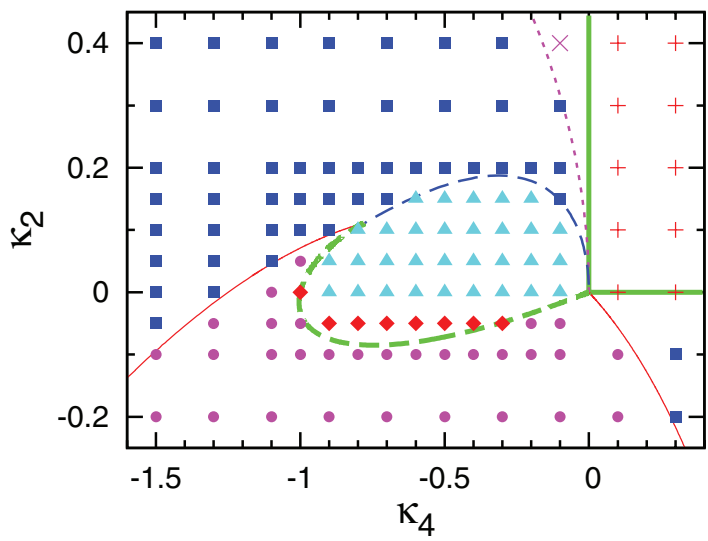

FIG. 1. (Color online) Dynamical phase diagram obtained numerically by solving Eqs. (12) and (13). In the region indicated by the plus, cross, and square symbols, a particle is motionless without deformations, with only 4-mode static deformation, and with 2- and 4-mode static deformations, respectively. The circles, triangles, and diamonds stand for a spinning motion, a standing oscillation, and an oscillation-spinning motion, respectively. The bifurcation lines are obtained analytically, whose derivations are described in the text.

of mass. In this spinning state, the shape of the particle is time independent in the co-rotating frame. The time series of the spinning state is displayed in Fig. 2(d) for $\kappa_{2}=-0.1$ and $\kappa_{4}=-0.1$ with the time interval $\Delta t=2.5$. In the region represented by the triangle, the particle with the 2- and 4-mode deformations undergoes a standing oscillation as shown in Fig. 3(a) for $\kappa_{2}=0$ and $\kappa_{4}=-0.1$ with the time interval $\Delta t=4$. Since this oscillation is a strong relaxation oscillation, we have shown a detailed time series with $\Delta t=0.6$ between $t=4$ and 8 and $t=8$ and 12 . In the region indicated by the diamond symbols, the oscillation-spinning motion appears, where the particle with the 2- and 4-mode deformations undergoes both standing oscillation and spinning motion. The
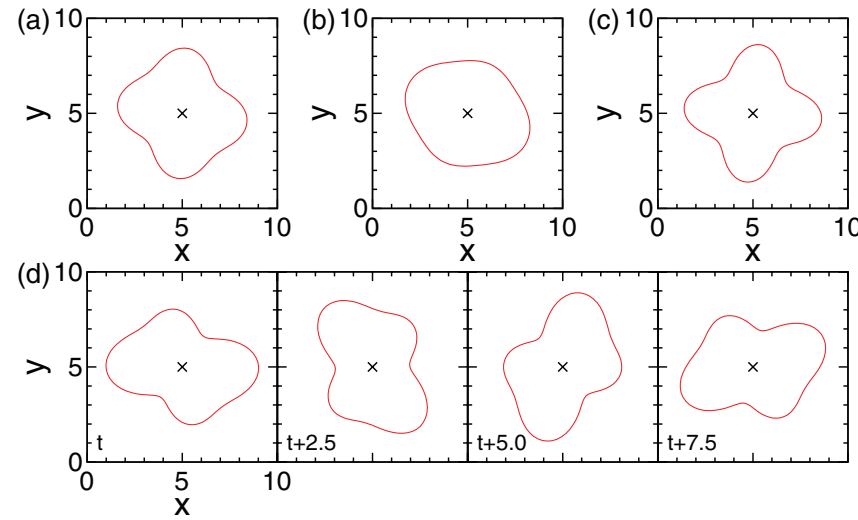

FIG. 2. (Color online) Snapshot of motionless static-deformed particle with 2- and 4-mode deformations for (a) $\kappa_{2}=0.2$ and $\kappa_{4}=-0.1$ and (b) $\kappa_{2}=-0.1$ and $\kappa_{4}=0.3$, and (c) with 4-mode deformation for $\kappa_{2}=0.4$ and $\kappa_{4}=-0.1$. (d) Time series of the snapshot of a spinning particle with 2- and 4-mode deformations for $\kappa_{2}=-0.1, \kappa_{4}=-0.1$ with time interval $\Delta t=2.5$, where the particle undergoes the clockwise rotation. The radius of a nondeformed circular shape is chosen as $R_{0}=3$. The center of mass is displayed by the cross. time series of this state is depicted in Fig. 3(b) for $\kappa_{2}=-0.05$ and $\kappa_{4}=-0.4$ with time interval $\Delta t=0.4$.

As mentioned in Sec. III, the coupled equations for the 2and 4-mode deformations have the same structure as those for 1 and 2 modes. In fact, we note that the motionless state with 2- and 4-mode static deformations, the spinning motion, the standing oscillation, and the oscillation-spinning motion correspond to the straight motion, the circular motion, the rectangular motion, and the quasiperiodic motion in the dynamic of the first and the second Fourier-mode deformations [28].

The lines in Fig. 1 are the stability limit obtained analytically in Sec. V. The thick solid line and the dotted line are the stability limit of the motionless circular particle without deformation and of the motionless particle with only 4-mode static deformation, obtained respectively from Eq. (35) and from Eq. (36). The thin solid lines are the bifurcation threshold between the motionless deformed state with both 2- and 4-mode static deformations and the spinning motion, given by Eq. (45). Here, note that we have checked numerically that this line coincides with the pitchfork bifurcation line obtained from $L_{\Theta}=0$ with $\operatorname{tr} L<0$ and $\operatorname{det} L>0$ for the set of solutions $\left(A_{2}, A_{4}, \Theta\right)$ given by Eqs. (37) and (38), and $\sin \Theta=0$. For this set of solutions, there is another bifurcation line displayed by the thin broken line in Fig. 1. This is the Hopf bifurcation line of the motionless deformed state with both 2-and 4-mode static deformations, given by $\operatorname{tr} L=0$ with $L_{\Theta}<0$ and $\operatorname{det} L>0$. Beyond this Hopf bifurcation, an oscillatory motion appears as predicted in Sec. V. The thick broken line is the Hopf bifurcation boundary of the spinning motion, which is obtained from Eq. (46). The corresponding bifurcation lines have been obtained for 1- and 2-mode equations in Ref. [28] except for the Hopf bifurcation line indicated by the thick broken line.

\section{DISCUSSION}

In this paper, we have investigated the dynamics of an active deformable particle whose shape deformation occurs spontaneously. The time-evolution equations for the Fourier components of deformations are constructed up to the 4-mode deformation and the couplings of different Fourier modes are considered up to the third orders for simplicity.

We have first shown that there exists a special symmetry between the coupled equations of $n$ - and $2 n$-mode deformations for different integers $n$. As an example, we have compared the dynamics obtained for the coupled time-evolution equations of the 1- and 2-mode deformations, and for the equations of the 2and 4-mode deformations. As a result, we have predicted by the theoretical analysis and confirmed by numerical simulations the existence of several types of solutions: the spinning motion of a deformed particle, the standing oscillation of deformations, and the oscillation-spinning motion, where both the oscillation of the deformation and the spinning motion occur, as well as the motionless particle with static deformations, for the equations of 2- and 4-mode deformations. Moreover, all the bifurcations of different solutions, except the bifurcation between the standing oscillation and the oscillation-spinning state, are obtained analytically, which are in good agreement with the numerical results as in Fig. 1. 

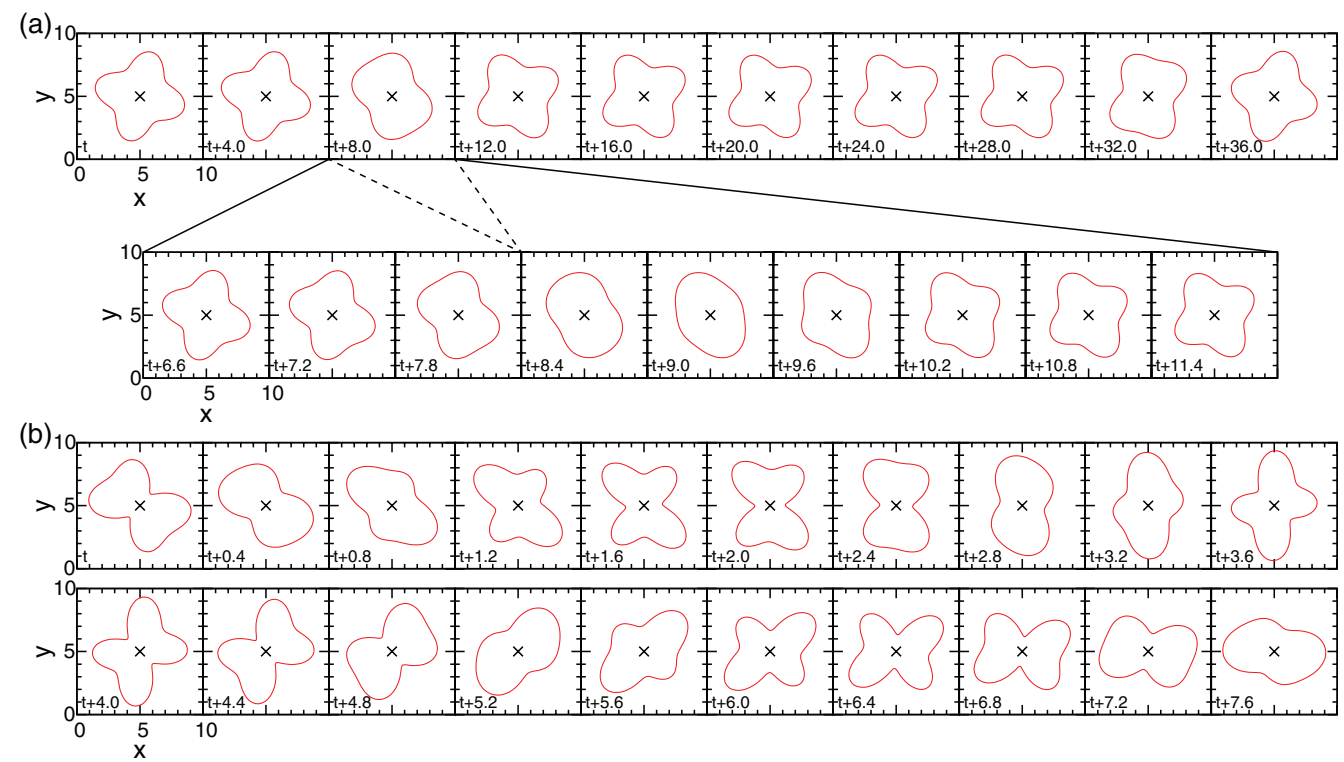

FIG. 3. (Color online) Time series of the snapshot of (a) standing oscillation of the 2- and 4-mode deformations for $\kappa_{2}=0$ and $\kappa_{4}=-0.1$ and (b) oscillation-spinning motion with 2- and 4-mode deformations for $\kappa_{2}=-0.05$ and $\kappa_{4}=-0.4$. The particle rotates in the clockwise direction. The time interval is chosen as $\Delta t=4$ for (a) and $\Delta t=0.4$ for (b). In Fig. (a), a detailed time series with $\Delta t=0.6$ is also shown for the time region where the motion is very rapid. After $t=7.6$, the shape returns to that $t=0$.

Actually, the coupling of the first and second mode deformations has been investigated in Refs. [27,28], where the variables are expressed in the form of a vector and a second rank tensor. In Appendix A, we summarize the relation between the Fourier components of the deformation and its tensor descriptions. Since the \pm 1 -mode Fourier components result in the displacement of the center of mass, they can be included in the velocity of the center of mass. Therefore, despite the mathematical equivalence of the time-evolution equations, the actual dynamics we have obtained in this paper for the time-evolution equations of the second and the fourth Fourier-mode deformations represents physically different behavior from those obtained in Refs. [27,28].

We make a remark about ignoring the $n=3$ mode in the present investigation. The results obtained for the 2- and 4-Fourier modes are justified as long as we are considering the situation that the center of mass of the particle is constant in time. In that case, we may put, as shown in Appendix B, all the modes with odd integers identically equal to zero.

The present results imply that there are at least two different mechanisms for a spontaneous spinning motion of a particle. One is the case where a particle generates an angular momentum. This has been modeled by introducing the antisymmetric tensor as a dynamic variable which couples with the migration velocity and the deformation tensors $[16,17,33]$. The other is the excitation of a propagating wave on the surface of a soft particle as described in the present paper. Then, a question arises as to how one can distinguish experimentally these two different kinds of spinning motions. We do not have any conclusive answer for this at present. But, one of the possibilities is to look for standing oscillation by changing the system parameters since such an oscillation never emerges in the spinning motion caused by the angular momentum. In fact, in Ref. [4], both a spontaneous spinning motion and a standing oscillation have been observed.

Finally, we briefly mention the dimensionality of space. In this paper, we have considered a spontaneous spinning motion only in two dimensions. In fact, most of the experiments of living cells such as protozoa are carried out on a substrate and hence, quasi-two-dimensional. However, there are many biological objects [10] and nonbiological droplets [34,35] which undergo a bulk three-dimensional self-propelled motion. Therefore, it is interesting to extend the present theory to three dimensions. However, a rotational motion in three dimensions is not necessarily axial symmetric. It has been shown that a helical motion appears as well as a circular motion [29]. Therefore the structure of bifurcations is expected to be more complex compared to two dimensions and systematic studies are needed. We shall return to this problem somewhere in the near future.

\section{ACKNOWLEDGMENTS}

This work was supported by the JSPS Core-to-Core Program "International research network for nonequilibrium dynamics of soft matter" and by a Grant-in-Aid for Scientific Research C (No. 23540449) from JSPS and a Grant-in-Aid for Scientific Research A (No. 24244063) from MEXT. M.T. is supported by the Japan Society for the Promotion of Science Research Fellowship for Young Scientists.

\section{APPENDIX A: TENSOR DESCRIPTIONS}

In this Appendix, we summarize the relation between the representations of deformations in two dimensions in terms of the Fourier components $c_{n}$ as Eq. (2) and in terms of the tensor variables as used in Refs. [16,17,27-31]. Tensor 
representation for deformations of a three-dimensional particle has been formulated by Fel [36].

For a second-mode deformation, we define the symmetric tensor of second rank by

$$
S_{i j}=\delta_{2} \sum_{m=1,2}\left(N_{i}^{(2, m)} N_{j}^{(2, m)}-\frac{1}{2} \delta_{i j}\right),
$$

with

$$
\boldsymbol{N}^{(n, m)}=\left(\cos \left(\theta_{n}+\frac{2 m}{n} \pi\right), \sin \left(\theta_{n}+\frac{2 m}{n} \pi\right)\right),
$$

where the angle $\theta_{n}$ is related with the Fourier components as Eq. (14). By setting $\delta_{2}=2 A_{2}$, we can write Eq. (A1) in terms of $c_{ \pm 2}$ as [31]

$$
S_{11}=-S_{22}=c_{2}+c_{-2}, S_{12}=S_{21}=i\left(c_{2}-c_{-2}\right) .
$$

In a similar way, a third-rank symmetric tensor is defined by

$$
U_{i j k}=\delta_{3} \sum_{m=1,2,3} N_{i}^{(3, m)} N_{j}^{(3, m)} N_{k}^{(3, m)} .
$$

By setting $\delta_{3}=(8 / 3) A_{3}$ we obtain the relation between $U_{i j k}$ and $c_{ \pm 3}$ as [31]

$$
\begin{aligned}
& U_{111}=-U_{122}=c_{3}+c_{-3}, \\
& U_{222}=-U_{112}=i\left(c_{3}-c_{-3}\right) .
\end{aligned}
$$

Here, due to the symmetry of the tensor $U$, the other components $U_{212}, U_{221}, U_{121}$, and $U_{211}$ are equated to $U_{122}$ (the former two) and $U_{112}$ (the latter two), respectively.

Finally, we show a symmetric-tensor representation of the fourth-mode deformation. The fourth-rank symmetric tensor is defined by

$$
\begin{aligned}
T_{i j k \ell}= & \delta_{4} \sum_{m=1}^{4}\left\{N_{i}^{(4, m)} N_{j}^{(4, m)} N_{k}^{(4, m)} N_{\ell}^{(4, m)}\right. \\
& \left.-\frac{1}{8}\left(\delta_{i j} \delta_{k \ell}+\delta_{i k} \delta_{j \ell}+\delta_{i \ell} \delta_{j k}\right)\right\} .
\end{aligned}
$$

By setting $\delta_{4}=4 A_{4}$, the relation between the symmetric tensor and $c_{ \pm 4}$ is given by $T_{1111}=T_{2222}=-T_{1122}=T^{+}$and $T_{1112}=$ $-T_{1222}=T^{-}$, where we have defined

$$
T^{+}=c_{4}+c_{-4}, T^{-}=i\left(c_{4}-c_{-4}\right) .
$$

The other components are obtained by the symmetric property of the tensor $T$ as $T_{1122}=T_{1212}=T_{1221}=T_{2112}=T_{2121}=$ $T_{2211}, \quad T_{1112}=T_{1121}=T_{1211}=T_{2111}, \quad$ and $T_{1222}=T_{2122}=$ $T_{2212}=T_{221}$.

\section{APPENDIX B: VELOCITY OF THE CENTER OF MASS}

The velocity of the center of mass can be written as [31]

$$
\boldsymbol{v}=\frac{1}{\Omega} \int d \omega V(\omega) \boldsymbol{R}(\omega),
$$

where $\Omega$ is the area of the particle and

$$
d \omega=R \sqrt{1+\left(R^{\prime} / R\right)^{2}} d \phi, \quad V=\frac{d R / d t}{\sqrt{1+\left(R^{\prime} / R\right)^{2}}},
$$

with $R^{\prime}=\partial R / \partial \phi$. The position vector $\boldsymbol{R}$ is given by a complex representation as $\boldsymbol{R}=R(\phi) e^{i \phi}$. Since $R(\phi)$ is given by Eq. (1) with (2) excluding $n= \pm 1$ modes, the integrand $d \omega V \boldsymbol{R}$ must take a factor $d \phi e^{i m \phi+i \phi}$ with $m$ even integer when $\delta R$ contains only the modes with even integers-in other words, the case that $m=-1$ is totally excluded. Therefore, after integral over $0<\phi<2 \pi$, the velocity of the center of mass $\boldsymbol{v}$ vanishes identically.
[1] K. Keren, Z. Pincus, G. M. Allen, E. L. Barnhart, G. Marriott, A. Mogilner, and J. A. Theriot, Nature (London) 453, 475 (2008).

[2] L. Bosgraaf and P. J. M. Van Haastert, PLoS ONE 4, e5253 (2009).

[3] L. Li, S. F. Nørrelykke, and E. C. Cox, PLoS ONE 3, e2093 (2008).

[4] Y. T. Maeda, J. Inose, M. Y. Matsuo, S. Iwaya, and M. Sano, PLoS ONE 3, e3734 (2008).

[5] T. Kaindl, H. Rieger, L.-M. Kaschel, U. Engel, A. Schmaus, J. Sleeman, and M. Tanaka, PLoS ONE 7, e42991 (2012).

[6] K. Nagai, Y. Sumino, H. Kitahata, and K. Yoshikawa, Phys. Rev. E 71, 065301(R) (2005).

[7] H. Boukellal, O. Campás, J.-F. Joanny, J. Prost, and C. Sykes, Phys. Rev. E 69, 061906 (2004).

[8] Y. Sumino, H. Kitahata, H. Seto, and K. Yoshikawa, Soft Matter 7, 3204 (2011).

[9] H. Wada and R. R. Netz, Phys. Rev. E 80, 021921 (2009).

[10] T. Ishikawa, J. R. Soc. Interface 6, 815 (2009).

[11] S. I. Nishimura, M. Ueda, and M. Sasai, PLoS Comput. Biol. 5, e1000310 (2009).
[12] S. Günther and K. Kruse, Europhys. Lett. 84, 68002 (2008).

[13] G. P. Alexander and J. M. Yeomans, Europhys. Lett. 83, 34006 (2008).

[14] D. Shao, W.-J. Rappel, and H. Levine, Phys. Rev. Lett. 105, 108104 (2010).

[15] F. Ziebert, S. Swaminathan, and I. S. Aranson, J. R. Soc. Interface 9, 1084 (2012).

[16] M. Tarama and T. Ohta, J. Phys.: Condens. Matter 24, 464129 (2012).

[17] M. Tarama and T. Ohta, Prog. Theor. Exp. Phys. (2013) 013A01.

[18] F. Takabatake, N. Magome, M. Ichikawa, and K. Yoshikawa, J. Chem. Phys. 134, 114704 (2011).

[19] W. L. Zeile, F. Zhang, R. B. Dickinson, and D. L. Purich, Cell Motil. Cytoskeleton 60, 121 (2005).

[20] H. C. Crenshaw, Amer. Zool. 36, 608 (1996).

[21] V. B. Shenoy, D. T. Tambe, A. Prasad, and J. A. Theriot, Proc. Natl. Acad. Sci. USA 104, 8229 (2007).

[22] W. R. DiLuzio, L. Turner, M. Mayer, P. Garstecki, D. B. Weibel, H. C. Berg, and G. M. Whitesides, Nature (London) 435, 1271 (2005). 
[23] K. Drescher, J. Dunkel, L. H. Cisneros, S. Ganguly, and R. E. Goldstein, Proc. Natl. Acad. Sci. USA 108, 10940 (2011).

[24] F.-L. Wen, K.-T. Leung, and H.-Y. Chen, Phys. Rev. E 86, 061902 (2012).

[25] D. Taniguchi, S. Ishihara, T. Oonuki, M. H.-Kitahata, K. Kaneko, and S. Sawai, Proc. Natl. Acad. Sci. USA 110, 5016 (2013).

[26] H. Ebata and M. Sano (unpublished).

[27] T. Ohta and T. Ohkuma, Phys. Rev. Lett. 102, 154101 (2009).

[28] T. Hiraiwa, M. Y. Matsuo, T. Ohkuma, T. Ohta, and M. Sano, Europhys. Lett. 91, 20001 (2010).

[29] T. Hiraiwa, K. Shitara, and T. Ohta, Soft Matter 7, 3083 (2011).
[30] M. Tarama and T. Ohta, Eur. Phys. J. B 83, 391 (2011).

[31] T. Ohta, T. Ohkuma, and K. Shitara, Phys. Rev. E 80, 056203 (2009).

[32] J. Guckenheimer and P. Holmes, Nonlinear Oscillations, Dynamical Systems, and Bifurcations of Vector Fields (SpringerVerlag, New York, 1983).

[33] R. Wittkowski and H. Löwen, Phys. Rev. E 85, 021406 (2012).

[34] T. Toyota, N. Maru, M. M. Hanczyc, T. Ikegami, and T. Sugawara, J. Am. Chem. Soc. 103, 5012 (2009).

[35] T. Ban, T. Yamagami, H. Nakata, and Y. Okano, Langmuir 29, 2554 (2013).

[36] L. G. Fel, Phys. Rev. E 52, 702 (1995). 